\title{
Spatially Regularized Common Spatial Patterns for EEG Classification
}

\author{
Fabien LOTTE, Cuntai GUAN \\ Institute for Infocomm Research $\left(I^{2} R\right)$, Singapore \\ fprlotte@i2r.a-star.edu.sg, ctguan@i2r.a-star.edu.sg
}

\begin{abstract}
In this paper, we propose a new algorithm for BrainComputer Interface (BCI): the Spatially Regularized Common Spatial Patterns (SRCSP). SRCSP is an extension of the famous CSP algorithm which includes spatial a priori in the learning process, by adding a regularization term which penalizes spatially non smooth filters. We compared SRCSP and CSP algorithms on data of 14 subjects from BCI competitions. Results suggested that SRCSP can improve performances, around 10\% more in classification accuracy, for subjects with poor CSP performances. They also suggested that SRCSP leads to more physiologically relevant filters than CSP.
\end{abstract}

\section{Introduction}

Brain-Computer Interfaces (BCI) are communication systems which enable users to send commands to computers by using brain activity only, this activity being generally measured by ElectroEncephaloGraphy (EEG) [8] . BCI are generally designed according to a pattern recognition approach, i.e., by extracting features from EEG signals, and by using a classifier to identify the user's mental state from such features $[8,6]$. The Common Spatial Patterns (CSP) algorithm is a feature extraction method which can learn spatial filters maximizing the discriminability of two classes [2]. CSP has been proven to be one of the most popular and efficient algorithms for BCI design, notably during BCI competitions [1].

However, CSP is known to be highly non-robust to noise and outliers $[4,10]$. Moreover, despite being a spatial filter, CSP completely ignores the spatial location of the EEG electrodes. To overcome these drawbacks, Grosse-Wentrup et al have proposed to use beamforming in order to learn CSP-like spatial filters which maximize the variance of the signals originating from a predefined Region Of Interest (ROI) while min- imizing the variance of the signals originating outside this ROI [4]. Despite the good results obtained, this method has a major limitation: it requires a strong a priori with the location and extent of the ROI involved.

In this paper, we propose a Spatially Regularized CSP (SRCSP) which incorporates spatial information in a generic way. As such, contrary to beamforming, SRCSP does not require additional a priori and can be used to classify any kind of mental states. SRCSP aims at regularizing the CSP objective function by penalizing filters which are not spatially smooth, i.e., for which neighboring electrodes have very different weights. Indeed, from a neurophysiological point of view, we expect neighboring electrodes to measure similar brain signals and as such to have a similar contribution in the spatial filter. It is expected that such a priori would help finding relevant spatial filters, even with noisy or few training EEG signals. Other domains also took advantage of spatial a priori $[11,3]$, which inspired the design of the proposed SRCSP.

This paper is organized as follows: Section 2 describes the CSP algorithm as well as the SRCSP algorithm we propose. Then, Section 3 describes the EEG data used for evaluation. Finally, Sections 4 and 5 present the results and conclude the paper, respectively.

\section{Methods}

\subsection{CSP algorithm}

CSP aims at learning spatial filters which can maximize the variance of band-pass filtered EEG signals from one class while minimizing the variance of EEG signals from the other class $[2,9]$. As the variance of EEG signals filtered in a given frequency band corresponds to the signal power in this band, CSP aims at achieving optimal discrimination for BCI based on band power features [9]. Formally, CSP uses the spatial filters $w$ which extremize the following function:

$$
J(w)=\frac{w^{T} X_{1}^{T} X_{1} w}{w^{T} X_{2}^{T} X_{2} w}=\frac{w^{T} C_{1} w}{w^{T} C_{2} w}
$$


where $T$ denotes transpose, $X_{i}$ is the data matrix for class $i$ (with the training samples as rows and the EEG electrodes as columns) and $C_{i}$ is the spatial covariance matrix of the EEG signals from class $i$, assuming a zero mean for the EEG signals. Note that this last assumption is generally met as EEG signals are usually bandpass filtered. This optimization problem is generally solved by jointly diagonalizing the two matrices $C_{1}$ and $\left(C_{1}+C_{2}\right)$ [2, 9]. An alternative but equivalent way to solve this optimization problem is obtained by first observing that the function $J(w)$ remains unchanged if the filter $w$ is rescaled. Indeed $J(k \times w)=J(w)$, with $k$ a real constant, which means the rescaling of $w$ is arbitrary. As such, extremizing $J(w)$ is equivalent to extremizing $w^{T} C_{1} w$ subject to the constraint $w^{T} C_{2} w=1$ as it is always possible to find a rescaling of $w$ such that $w^{T} C_{2} w=1$. Using the Lagrange multiplier method, this constrained optimization problem amounts to extremizing the following function:

$$
L(\lambda, w)=w^{T} C_{1} w-\lambda\left(w^{T} C_{2} w-1\right)
$$

The filters $w$ extremizing $L$ are such that the derivative of $L$ with respect to $w$ equals 0 :

$$
\begin{aligned}
\frac{\partial L}{\partial w} & =2 w^{T} C_{1}-2 \lambda w^{T} C_{2}=0 \\
& \Leftrightarrow C_{1} w=\lambda C_{2} w \\
& \Leftrightarrow \quad C_{2}^{-1} C_{1} w=\lambda w
\end{aligned}
$$

We then obtain a standard eigenvalue problem. Therefore, the spatial filters extremizing Equation 1 are the eigenvectors of $M=C_{2}^{-1} C_{1}$ which correspond to its largest and lowest eigenvalues.

\subsection{Spatially Regularized CSP algorithm}

In this paper we propose the SRCSP algorithm, in order to include spatial information into the learning process of the CSP. More particularly, we would like to obtain spatially smooth filters $w$. Indeed, as mentioned earlier, from a neurophysiological point of view, neighboring neurons tend to have similar functions, which supports the idea that neighboring electrodes should measure similar brain signals (if the electrodes are close enough to each other). To ensure spatial smoothness of the filters $w$, we add a penalty term $P(w)$ to the objective function $J(w)$ of the classical CSP:

$$
J_{P}(w)=\frac{w^{T} C_{1} w}{w^{T} C_{2} w+\alpha P(w)}
$$

$P(w)$ is a function measuring the spatial smoothness of the spatial filters $w$. The spatially smoother $w$, the lower $P(w)$. Therefore, to maximize $J_{P}(w)$, we need to minimize $P(w)$, hence ensuring spatially smooth filters. The variable $\alpha$ is a user-defined positive constant which defines the desired level of filter smoothness (the higher $\alpha$, the spatially smoother the filters). Here, we use a Laplacian penalty of the form $P(w)=w^{T} K w$ with $K=D-G$, as in [11]. $G$ is a Gaussian kernel such that $G_{i j}=\exp \left(-\frac{1}{2} \frac{\left\|v_{i}-v_{j}\right\|^{2}}{r^{2}}\right)$, with $v_{i}$ being the 3D coordinates of the $i^{t h}$ electrodes. The hyperparameter $r$ defines how far two electrodes can be to be still considered as close to each other. $D$ is a diagonal matrix such as $D_{i i}=\sum_{j} G_{i j}$. As $w^{T}(D-G) w=\frac{1}{2} \sum_{i, j} G_{i j}\left(w_{i}-\right.$ $\left.w_{j}\right)^{2}$ (see, e.g., [3]), this term will be large for nonsmooth filters, i.e., for filters in which neighboring electrodes have very different weights. With such a penalty term, Equation 3 becomes:

$$
\begin{aligned}
J_{P}(w) & =\frac{w^{T} C_{1} w}{w^{T} C_{2} w+\alpha w^{T} K w} \\
& =\frac{w^{T} C_{1} w}{w^{T}\left(C_{2}+\alpha K\right) w}
\end{aligned}
$$

In this case, the Lagrangian is as follows:

$$
L_{P}(\lambda, w)=w^{T} C_{1} w-\lambda\left(w^{T}\left(C_{2}+\alpha K\right) w-1\right)
$$

By following the same approach as previously (see Section 2.1), we obtain the following eigenvalue problem:

$$
\left(C_{2}+\alpha K\right)^{-1} C_{1} w=\lambda w
$$

Thus, maximizing $J_{P}(w)$ consists in taking the eigenvectors corresponding to the largest eigenvalues of $M_{1}=\left(C_{2}+\alpha K\right)^{-1} C_{1}$ as the filters $w$. With CSP, the eigenvectors corresponding to both the largest and smallest eigenvalues of $M$ (see Section 2.1) are taken as spatial filters, as they respectively maximize and minimize Equation 1 [2]. However, for SRCSP, we cannot use the eigenvectors corresponding to the lowest eigenvalues of $M_{1}$, as they will minimize Equation 3, and as such will maximize the penalty term, i.e., they will lead to highly non smooth filters. Therefore, in order to obtain the filters which maximize $C_{2}$ while minimizing $C_{1}$, we also need to maximize the following objective function, which is the counterpart of Equation 3:

$$
J_{P}(w)=\frac{w^{T} C_{2} w}{w^{T} C_{1} w+\alpha P(w)}
$$

which is simply achieved by taking the eigenvectors corresponding to the largest eigenvalues of $M_{2}=\left(C_{1}+\right.$ $\alpha K)^{-1} C_{2}$ as the filters $w$. In other words, with SRCSP, the spatial filters used are the eigenvectors corresponding to the largest eigenvalues of $M_{1}$ and to the largest eigenvalues of $M_{2}$. 
SRCSP has two hyperparameters: $r$ which defines the size of the neighborhood considered for smoothing, and $\alpha$ which defines the level of spatial smoothness the filters should reach. In this work, we used as their optimal values, the ones which maximized the 10 -fold cross validation accuracy on the training set, by using Linear Discriminant Analysis (LDA) [5] as the classifier.

\section{EEG data sets for evaluation}

In order to assess the proposed SRCSP, we compared it with the classical CSP approach on EEG data of 14 subjects, from 2 publicly available data sets of BCI competitions. They are described below.

\subsection{Data set IVa, BCI competition III}

Data set IVa, from BCI competition III [1], contains EEG signals from 5 subjects, who performed right hand and foot Motor Imagery (MI), i.e., imagination of right hand and foot movements [8]. EEG were recorded using 118 electrodes. A training set and a testing set were available for each subject. The size of these sets was different for each subject. More precisely, 280 trials were available for each subject, among which 168, 224, 84, 56 and 28 composed the training set for subject A1, A2, A3, A4 and A5 respectively, the remaining trials composing the test set.

\subsection{Data set IIa, BCI competition IV}

Data set IIa from BCI competition IV $^{1}$ (provided by the Graz group [7]) comprises EEG signals from 9 subjects who performed left hand, right hand, foot and tongue MI. The EEG signals were recorded using 22 electrodes. For the purpose of this study, only EEG signals corresponding to left and right hand MI were used. A training and a testing set were available for each subject, both sets containing 72 trials for each class.

For both data sets, EEG signals were band-pass filtered in the $8-30 \mathrm{~Hz}$ frequency band using a $5^{\text {th }}$ order Butterworth filter. Indeed, this frequency band contains the main frequencies involved in MI [8]. In this work, we considered the discrete classification of the trials, i.e., we assigned a class to each trial and not to each trial sample. For both data sets, for each trial, we extracted the features from the time segment located from $0.5 \mathrm{~s}$ to $2.5 \mathrm{~s}$ after the cue instructing the subject to perform MI (as done by the winner of BCI competition IV, data set

\footnotetext{
1http://ida.first.fhg.de/projects/bci/ competition\_iv/
}

2a). With both CSP and SRCSP, we used the 3 most discriminative pairs of filters for feature extraction, as recommended in [2]. In other words, we used the filters corresponding to the 3 largest and 3 smallest eigenvalues of $M$ for CSP, and the filters corresponding to the 3 largest eigenvalues of $M_{1}$ and the 3 largest eigenvalues of $M_{2}$ for SRCSP (See Section 2).

\section{Results and discussion}

For each subject, the CSP/SRCSP filters were learnt on the training set available. The log-variances of the spatially filtered EEG signals were then used as the input features to an LDA classifier, one of the most popular and efficient classifier for BCI design [5]. Table 1 report the classification accuracies obtained when classifing the EEG signals from the test sets.

On average, SRCSP gave better classification accuracies (mean: $78.7 \pm 13.9$ ) than CSP (mean: $73.1 \pm$ 17.6). However, this difference is not significant at the 5\% level (but nearly: $p=0.07$ with a two-tailed paired t-test), which might be due to the sample size. Nevertheless, results suggest that SRCSP does increase the classification accuracy specifically for subjects with poor CSP performances but not for those with already good performances. Indeed, for subjects with poor CSP accuracies (e.g., A3, A5, B2 and B5), using SRCSP leads to dramatic increase in performance, typically a gain of classification accuracy of $10 \%$ or more, whereas for already good subjects (e.g., A2, B1, B2, B8 and B9) using SRCSP leads to similar performances than CSP.

Interestingly enough, with CSP, subjects A3, A5, B2 and B5 would have been identified as "BCI illiterates", meaning they cannot use a BCI, as their performances were close to random (here below 55\%). With SRCSP, none of these subjects would be identified as illiterates anymore. The most dramatic increase in performance was for subject A5, who gained $37 \%$ of accuracy. It should be noted that this subject was the one with the smallest training set (only 28 trials). Thus, we might hypothesize that adding an a priori to the algorithm, here a spatial one, can actually help by guiding the optimization process to find good spatial filters despite the limited amount of training data. In summary, it seems that SRCSP may learn good spatial filters even when training EEG data are limited or noisy, thanks to a regularization based on a spatial a priori.

Figure 1 shows some examples of spatial filters obtained with both CSP and SRCSP. As expected by adding the spatial regularization, the SRCSP filters are indeed smoother than the CSP filters. More interestingly, it also shows that SRCSP filters are more convincing from a neurophysiological point of view. For 
Table 1. Classification accuracies (\%) obtained for each subject for both the CSP and the proposed SRCSP method. For each subject, the best result is displayed in bold characters.

\begin{tabular}{|c||c|c|c|c|c||c|c|c|c|c|c|c|c|c||c|}
\hline \multicolumn{1}{|c||}{} & \multicolumn{4}{c||}{ BCI competition III, data set IVa } & \multicolumn{1}{c||}{ BCI competition IV, data set IIa } & \\
\hline Subject & A1 & A2 & A3 & A4 & A5 & B1 & B2 & B3 & B4 & B5 & B6 & B7 & B8 & B9 & Mean \\
\hline CSP & 66.07 & 96.43 & 47.45 & 71.88 & 49.6 & 88.89 & 51.39 & 96.53 & $\mathbf{7 0 . 1 4}$ & 54.86 & $\mathbf{7 1 . 5 3}$ & $\mathbf{8 1 . 2 5}$ & 93.75 & $\mathbf{9 3 . 7 5}$ & $73.1 \pm 17.6$ \\
\hline SRCSP & $\mathbf{7 2 . 3 2}$ & 96.43 & $\mathbf{6 0 . 2}$ & $\mathbf{7 7 . 6 8}$ & $\mathbf{8 6 . 5 1}$ & 88.89 & $\mathbf{6 3 . 1 9}$ & 96.53 & 66.67 & $\mathbf{6 3 . 1 9}$ & 63.89 & 78.47 & $\mathbf{9 5 . 8 3}$ & 92.36 & $\mathbf{7 8 . 7} \pm \mathbf{1 3 . 9}$ \\
\hline
\end{tabular}

instance, for subject A5, the CSP filter appear as very rough and noisy, with large weights in many different electrode locations, whereas the corresponding SRCSP filter displays large weights in a few regions, located over the motor cortex of the brain, which is expected from the neurophysiology literature [8]. Thus, this suggests that SRCSP may lead to more physiologically relevant, and thus more interpretable filters than CSP.

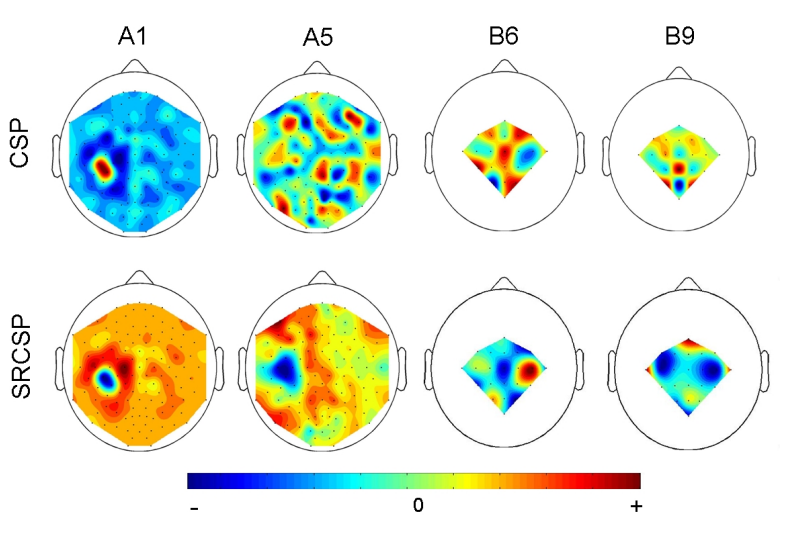

Figure 1. Electrode weights for corresponding CSP and SRCSP filters, for subjects $A 1, A 5$ (both using 118 electrodes) and B6, B9 (both using 22 electrodes).

\section{Conclusion}

In this paper, we proposed the Spatially Regularized Common Spatial Pattern (SRCSP) algorithm, an extension of the famous CSP which includes spatial a priori into the learning process. To do so, we added a regularization term to the CSP objective function to ensure spatially smooth filters, and we derived an algorithm to optimize this new objective function. We compared the SRCSP and the CSP algorithms on EEG data of 14 subjects from BCI competition data sets. Results suggested that SRCSP could lead to large performance improvements for subjects with initially poor CSP accuracies, while giving similar performances than CSP for subjects with already good performances. Our results also suggested that SRCSP may lead to more physiologically relevant filters than CSP. To conclude, we would recommend adopting SRCSP instead of CSP for BCI design, especially for subjects with few and noisy data. Acknowledgments: The authors would like to thank Dr. Blankertz for providing the electrodes coordinates of BCI competition data, and Dr. Hamadicharef for his constructive comments on this manuscript.

\section{References}

[1] B. Blankertz et al. The BCI competition III: Validating alternative approaches to actual BCI problems. IEEE Trans. on Neural Sys. and Rehab., 14(2):153-159, 2006.

[2] B. Blankertz, R. Tomioka, S. Lemm, M. Kawanabe, and K.-R. Müller. Optimizing spatial filters for robust eeg single-trial analysis. IEEE Signal Proc Magazine, 25(1):41-56, 2008.

[3] D. Cai, X. He, Y. Hu, J. Han, and T. Huang. Learning a spatially smooth subspace for face recognition. In CVPR, 2007.

[4] M. Grosse-Wentrup, C. Liefhold, K. Gramann, and M. Buss. Beamforming in noninvasive braincomputer interfaces. IEEE Trans. on Biomed. Eng., 56(4):12091219,2009

[5] F. Lotte, M. Congedo, A. Lécuyer, F. Lamarche, and B. Arnaldi. A review of classification algorithms for EEG-based brain-computer interfaces. J. of Neural Eng., 4:R1-R13, 2007.

[6] F. Lotte, H. Mouchère, and A. Lécuyer. Pattern rejection strategies for the design of self-paced EEG-based braincomputer interfaces. In ICPR, pages 1-5, 2008.

[7] M. Naeem, C. Brunner, R. Leeb, B. Graimann, and G. Pfurtscheller. Seperability of four-class motor imagery data using independent components analysis. J. of Neural Eng., 3:208-216, 2006.

[8] G. Pfurtscheller and C. Neuper. Motor imagery and direct brain-computer communication. proc. of the IEEE, 89(7):1123-1134, 2001.

[9] H. Ramoser, J. Muller-Gerking, and G. Pfurtscheller. Optimal spatial filtering of single trial EEG during imagined hand movement. IEEE Trans. on Rehab., 8(4):441446, 2000.

[10] B. Reuderink and M. Poel. Robustness of the common spatial patterns algorithm in the BCI-pipeline. Technical report, HMI, University of Twente, 2008.

[11] Z. Xiang, Y. Xi, U. Hasson, and P. Ramadge. Boosting with spatial regularization. In NIPS, 2009. 